\title{
An Overview on Researches on Underwater Sensor Networks: Applications, Current Challenges and Future Trends
}

\author{
Pedram Vahdani Amoli \\ Department of Computer Engineering, College of Engineering, Tehran North Branch, Islamic Azad University, \\ Tehran, Iran
}

\section{Article Info \\ Article history: \\ Received Oct 14, 2015 \\ Revised Jan 21, 2016 \\ Accepted Feb 4, 2016}

\section{Keyword:}

Target tracking

Underwater sensor networks UWSN

\begin{abstract}
Nowadays, Underwater Sensor Networks (UWSN), in which data is collected through underwater sensors, have drawn lots of concern. Generally, wireless sensor network (WSNs) have important applications such as remote environmental monitoring and target tracking. This possibility is enabled by the available smaller and cheaper sensors. These sensors are equipped with wireless interfaces which they form a network. However, there are various problems specified to underwater environments, including the communication medium. Designs of these types of networks significantly depend on their application, and factors such as environment, design objectives of the application, cost, hardware and system constraints. The goal of this work is to review the literature on various aspects of UWSNs, and present an overview of several new applications and their challenges. Publications are reviewed to show the statistics of published works in several aspects of the topic based upon the year of publication. This survey gives the readers a view on the place of underwater sensor networks on researches and industries. The readers can track what have been highly interests in recent years and what are yet on challenges.
\end{abstract}

Copyright (C) 2016 Institute of Advanced Engineering and Science. All rights reserved.

\section{Corresponding Author:}

Pedram Vahdani Amoli,

Departement of Computer Engineering, College of Engineering,

Tehran North Branch, Islamic Azad University,

No.79, Ghobadian Aly, Next to Mirdamad Blvd, Tehran, Iran.

Email: pedramvahdani@gmail.com

\section{INTRODUCTION}

Nowadays, Underwater Sensor Networks (UWSN) is of high concern in a variety of fields such as industry, science, military, and so on. Today, majority of underwater communication systems works with acoustic technology. Factors that influence acoustic communications are path loss, noise, multi-path, delay, dropper spread.

Typically, some sensors in UWSNs send their observed data to sink by multi-hop communication, but since building this type of network is in the water, therefore applying the terrestrial network protocol is difficult [1],[2]. For example, in terrestrial network, they used radio or optical waves for wireless communication. But in UWSN, it is hard to use these medium, hence they used acoustic waves. It is essential for acoustic communication to consider long delay problems that are mainly caused by the long propagation delay. Bandwidth limitation and high bit error rate, power limitation, sensor failures are the challenging problems for UWSN due to its challenging environment. Under Water Sensor Networks has two-dimensional and three dimensional architecture [1].

In this paper, a brief review has been made on Underwater Sensor Networks, its applications and their strength and weaknesses. Then brief statistics of UWSN has been reviewed and finally it was compared with terrestrial sensor networks and the differences are highlighted. 


\section{LITERATURE REVIEW}

Sensing and subsequent transmission of radio frequency waves in sub-sea environment and deep sea exploration requires a particular approach for medium in communicating. Noticing the fact that a huge amount of unexploited resources lies in the $70 \%$ of the earth covered by oceans this technology is mode more apparently critical to the new world. Yet, the aquatic world has mainly been unaffected by the recent advances in the area of wireless sensor networks and their pervasive penetration in modern day research and industrial development. However, majority of underwater deployments rely on acoustics for enabling communication combined with special sensors having the capacity to take on harsh environments of the oceans. A recently published survey paper presented by Murad et al. [3] particularly focuses on gathering most recent developments and experimentation related to key underwater sensor network applications and acoustic-type underwater sensor networks deployments for monitoring and controlling of underwater domains.

Since sensing and subsequent transmission and radio frequency waves tend to vary for different subsea environments, in another work of the same authors, Felemban et al. [4] has presented a survey paper focusing on gathering most recent developments in underwater sensor networks applications and their deployments. In that paper, the authors have classified the underwater applications into five main classes as monitoring, disaster, military, navigation, and sports, to cover the large spectrum of these sensor networks. These applications are further divided into relevant subclasses. They have also shown the challenges and opportunities faced by recent deployments of underwater sensor networks.

Node deployment is task for acoustic underwater network that effects network topology control, routing, and boundary detection [5]. 2-D terrestrial wireless sensor networks have been studied much more than 3-D counterparts but Han et al. recently published a work on the impacts of node deployment strategies on localization performances in a 3-D environment [5]. Simulation experiment in that work reveals that the regular tetrahedron deployment scheme has a better result in terms of reducing localization error and increasing localization ratio compare to the random deployment scheme and the cube deployment scheme.

Earthquake and tsunami forewarning, naval surveillance, and marine biology are examples of important applications in underwater acoustic sensor networks. As the GPS signal is highly absorbed within the water, whereas radio frequency signals attenuate more. When optical signals propagate within the underwater environment they scatter. On the contrary, acoustic signals are preferred for communication between the underwater sensor nodes.

The location information of sensor nodes is important and they should be accompanied with data collected through sensors. Thus, localization for underwater acoustic sensor network has becomes a very interesting topic of research in the last decade. However, several limitations of acoustic channel like low bandwidth, high bit error rate etc. along with long propagation delay of the sound wave and limited battery power of the sensor nodes within the water make the localization of underwater nodes very challenging. Regarding these challenges, an event-driven time-synchronization free distributed localization scheme for large scale three dimensional underwater acoustic sensor networks is presented in the work of Kundu and Sadhukhan [6]. Their scheme employs a recursive localization process in which successfully localized nodes can act as reference node to aid for localization of the other ordinary sensor nodes and two ways for distance measurement in order to avoid the requirement of time synchronization.

Underwater acoustic sensor node cannot rely on the GPS to position itself, and the traditional indirect positioning methods used in Ad Hoc network is not fully applicable to the localization of underwater acoustic sensor networks. Hence, an improved underwater acoustic network localization algorithm is needed. This algorithm should be enabled to process the raw data before localization calculation to enhance the tolerance of random noise. $\mathrm{Wu}$ and $\mathrm{Li}$ in thier recent work introduced such a method which reduces the redundancy of the calculation results by using a more accurate basic algorithm and an adjusted calculation strategy [7].

Setting parameters in UWSN is necessary for having efficiency, therefore different MAC protocols are needed for different tasks. For acoustic UWSN localization it is a vital task which requires multiple packet exchanges. Ramezani and Leus concerned the problem of designing a MAC protocol for an underwater acoustic sensor network which efficiently schedules the localization packets of the anchors [8]. Scheduling protocol can minimized propagation delay for localization duration by knowing anchors position and their maximum transmission ranges. In that work concept of formulating collision-free packet transmission for localization has been done in order to know how an optimum solution can be obtained. In addition, they modeled the problem as a mixed integer linear program both in single-channel and multichannel scenarios. Furthermore, they proposed two algorithms with low-complexity and they compare it with the optimal solution as well as other existing methods. Based on simulation result their proposed method has shown performance close to optimum [8]. 
Generally challenges in underwater acoustic sensor networks include gradual propagation of acoustic waves, bounded bandwidth, immense and irregular propagation delay, ambient noise and transmission loss. Krishnaswamy and Manvi [9] have analyzed sound speed and transmission loss of acoustic channels using MATLAB simulation. They analyzed parameters of absorption coefficient, propagation delay and sound speed at various depth and transmission loss [9].

In general, battery-powered sensors in a sensor network operates while they can. In this context, it has two competing objectives; the first one is to maximized performance based on upper bound probability of successful search for false alarms, and second one is to maximized network operational time. As both sensing and communication of data use battery energy thus accurate amount of energy is needed to improve the operational lifetime of the sensor network. In a recently published paper, Jha et al. [10] presents an optimal energy allocator for nodes to manage energy consumption adaptable according to that sensing and communication node to maximize the network performance subject to specified constraints. Fixed total amount of energy allocation for specific time reduce the problem to synthesis an optimal network topology that maximizes successful search probability in a surveillance region. In the work of Jha et al. [10] a twostage optimization was used for an optimal solution. Adaptation to energy variations across the network could change by using sensing and communication models for underwater environment. Pareto optimal obtained trade-off between network lifetime and probability of successful search over surveillance area [10].

While monitoring UWSN in a given area performs by number of sensors. Designing energyefficient routing protocols became essential for sensor nodes that in fact powered by batteries and operate in an underwater environment which is harsh and its propagation delay is long. Majority of routing protocols uses greedy approaches to deliver data. One of these approach is depth based routing, DBR. Further, this routing protocol requires only local depth information which can easily obtained by an inexpensive depth sensor. DBR uses smaller depth as the only metric for choosing a route; however it might result in high energy consumption and a long delay that would degrade network performance. To address this problem, Mohammadi et al. [11] proposed an improved DBR protocol by using routing based on remaining energy of receiver node in conjunction with the depth difference of receiver node and previous forwarder node and the number of hops that a packet has traveled. Their simulation was carried out in Aquasim an NS2 based underwater simulator. The comparison between DBR and Fuzzy multi metric DBR protocol has shown that FDBR has outperforms DBR in terms of average end to end delay, packet delivery ratio and energy saving [11].

Long acoustic propagation delay, limited resources and water currents are the major challenges for energy-efficient and low-latency routing protocols of UWSN. As sensors always move with currents consequently network, topology of underwater sensor networks becomes dynamic and complex. For solving this problem adoptable geographic protocols has been proposed but localization in three-dimensional is hard to obtain. As depth-based routing protocol (DBR) uses easier way to get information by using depth information which in fact it is more practical for underwater sensor networks. Yet, it just not enough information for forwarding packet. Truthfully, it cause energy waste and increase end-to-end delay triggered by multiple forwarding paths of a packet. To settle this problem a new technique has been presented by Diao [12] that introduce underwater time of arrival (ToA) ranging technique. In addition they have made following changes: energy-efficient depth-based routing protocol that reduces redundancy energy cost in some blind zones; low-latency depth-based routing protocol that is able to deliver a packet through an optimal path.

In underwater acoustic, transmitting and communication directly effects energy consumption. Venkatesan and Li [13] have studied square grid topology for two-dimensional deployment strategy of underwater sensor networks. They have introduced a mathematical model to investigate the deployment error of underwater sensor networks. According to their experiment their model, with a new introduced parameter, has better robustness and has balanced energy consumption [13].

Due to vulnerability of underwater acoustic sensor networks to malicious attacks and due to their communication channel, urge of secure communication has raised. This need entails rapid development of secure communication mechanisms for underwater sensor nodes. In recent work of Han et al. [14], they presented a survey of secure communications in underwater sensor networks.

In order to have efficient routing protocols for data packet delivery in underwater sensor networks (UWSNs) and deal with roughness of acoustic channel network, coding has become vital. This technique is promising technique for efficient data packet delivery because of acoustic channels broadcast nature and their sensor nodes high computation capabilities. In this work, Hao et al. [15] introduced GPNC which is a geographic routing protocol for underwater sensor networks that works cooperatively with partial network coding to encode data packets and forward data to sink node. They have mentioned that GPNC has effectively reduced delays and retransmission which caused additional energy uses. The simulation results 
given in the work of Hao et al. [15] showen that this new method can incredibly improve performance and packet delivery ration while comparing with other routing protocols.

MTC or Localizing machine-type communication devices or sensors is an important issue in UWSN because of their increasing popularity of machine-to-machine (M2M) communication networks for locationbased applications. By implementing MTC with efficient localization, error rate and energy consumption of MTC devices can reduce. Since sensors has been used as an integral part of M2M communication networks which achieved popularity in underwater applications, further research has been conducted on sensor localization in both underwater and terrestrial M2M networks. The main challenges of designing an underwater localization are the lack of good radio signal propagation in underwater, sensor mobility management, and ensuring network coverage in 3D underwater M2M networks. Moreover, predicting the mobility pattern of MTC devices, trading-off energy consumption and location accuracy are another major challenges of designing terrestrial localization techniques. Karim et al. [16] presents a survey on MTC for both terrestrial and underwater localization approaches based on current researches. They have also classified localization approaches based on several factors, and identified their limitations with potential solutions, and compare with them [16].

S. D. Seeley Jr. et al. [17] Introduced software for android users that improved existing AUV (Autonomous Underwater Vehicles) mission planner, VectorMap, which has used Google maps to enhance mobile technology in field of UWSN. In the work of H. Mei et al. [18] has propose a two-step approach to improve performance of CFO (carrier frequency offset) so OFDM (frequency-division-multiplexing) would be handled better. This has been achieved through reducing BER. They have mentioned CS-based channel has better performance than that LS channel.

\subsection{Research trends and statistics in literature}

Underwater sensor networks as a topic of interest has been attractive for researchers and industries. Based on brief review on the literature which has been made through Google Scholar, the statistics on these topics have been summarized in Table 1 . Table 1 lists a number of attractive applications of underwater sensor networks for their topics and their usage. Research statistics of this table shows researchers interests for a decade.

Table 1. Research topics of interest in the last 10 years (2005-2015) on UWSN (Y: Yes)

\begin{tabular}{|c|c|c|c|c|c|}
\hline Topics & Usage & $\begin{array}{c}\text { Examples of literatures } \\
\text { on topic }\end{array}$ & $\begin{array}{c}\text { Current } \\
\text { Use/Research }\end{array}$ & $\begin{array}{c}\text { Current } \\
\text { Challenge? }\end{array}$ & $\begin{array}{c}\text { Future } \\
\text { Trends? }\end{array}$ \\
\hline Mine countermeasure & Military & {$[2,18,21,22]$} & $\mathrm{Y}$ & $\mathrm{Y}$ & $\mathrm{Y}$ \\
\hline Barrier reef & Military & {$[3,4,23]$} & $\mathrm{Y}$ & $\mathrm{Y}$ & $\mathrm{Y}$ \\
\hline Equipment monitoring & Military/ Monitoring & {$[1,2,3-4,19-20,23]$} & $\mathrm{Y}$ & $\mathrm{Y}$ & $\mathrm{Y}$ \\
\hline Autonomous surveillance & Military & {$[2,20,22,24]$} & $\mathrm{Y}$ & $\mathrm{Y}$ & $\mathrm{Y}$ \\
\hline Marine fish farms & Monitoring & [25] & $\mathrm{Y}$ & $\mathrm{Y}$ & $\mathrm{Y}$ \\
\hline Offshore platform protection & Monitoring/ Disaster & {$[2,3,4,22]$} & $\mathrm{Y}$ & $\mathrm{Y}$ & $\mathrm{Y}$ \\
\hline Resource exploration & Monitoring & {$[1,2,3-4,19-21,23]$} & $\mathrm{Y}$ & $\mathrm{Y}$ & $\mathrm{Y}$ \\
\hline Navigation & Military/ Navigation & {$[1,2,3-4,19-21,23]$} & $\mathrm{Y}$ & $\mathrm{Y}$ & $\mathrm{Y}$ \\
\hline Communication & Military/ Navigation & {$[1,2,3-4,19-21,23]$} & $\mathrm{Y}$ & $\mathrm{Y}$ & $\mathrm{Y}$ \\
\hline
\end{tabular}

\section{RESEARCH TRENDS AND STATISTICS}

Several research works in UWSN have been reviewed through Google Scholar in order to list a categorical table for underwater sensor networks. This review is made from the year 2000 to now. Research statistics on these aspects on the UWSNs has shown an increase on published works and also interest of the researchers in recent years.

It should be noted that the statistics on 2014-2015 works are not the finals since the works publishing on the coming few months and also those paginated in the early 2016 may still counted as the statistics of 2014-2015. Reports given in Table 2 present an estimate on the researchers' interests and which category has more published papers. Table 3 gives an estimate on the number of published works in the journals and proceedings separated by years and total counts. 
Table 2. Statistics on categories of underwater sensor networks reseach (Ref: Google scholar \& IEEE)

\begin{tabular}{lccccc}
\hline \multicolumn{1}{c}{ Category } & $2000-2007$ & $2008-2009$ & $2010-2011$ & $2012-2013$ & $2014-2015$ \\
\hline Acoustic underwater sensor networks & 57 & 83 & 107 & 133 & $\sim 79$ \\
Wireless underwater sensor networks & 17 & 46 & 72 & 83 & $\sim 80$ \\
Routing & 12 & 27 & 38 & 56 & $\sim 56$ \\
Energy efficiency and consumption & 6 & 20 & 30 & 30 & $\sim 25$ \\
Localization & 11 & 25 & 30 & 40 & $\sim 30$ \\
Survey on UWSN & 1 & 6 & 4 & 9 & $\sim 11$ \\
Sensors communication & 6 & 12 & 19 & 9 & $\sim 10$ \\
Sensor deployment & 3 & 10 & 8 & 11 & $\sim 9$ \\
Sensor positioning & 5 & 1 & 3 & 5 & $\sim 3$ \\
Mobile sensors & 4 & 10 & 11 & 18 & $\sim 19$ \\
\hline
\end{tabular}

Table 3. Statistics on scientific journals publishing on underwater sensor networks

\begin{tabular}{|c|c|c|c|c|c|c|c|}
\hline Journal/Proceedings & Publisher & $2000-2007$ & $2008-2009$ & $2010-2011$ & $2012-2013$ & $2014-2015$ & Total \\
\hline IEEE Supported Conferences & IEEExplore & 165 & 93 & 98 & 99 & 98 & 553 \\
\hline Hindawi Journals & Hindawi & 0 & 0 & 5 & 9 & 19 & 33 \\
\hline Proceedings of Intl. Conference & $\begin{array}{l}\text { ACM Digital } \\
\text { Library }\end{array}$ & 3 & 4 & 2 & 14 & 8 & 41 \\
\hline Computer Communications & Elsevier & 0 & 2 & 0 & 3 & 1 & 6 \\
\hline Ad hoc Networks & Elsevier & 2 & 3 & 4 & 4 & 6 & 19 \\
\hline IEEE Journals & IEEE & 45 & 14 & 27 & 28 & 40 & 154 \\
\hline Systems & John Wiley & 0 & 0 & 0 & 0 & 4 & 4 \\
\hline
\end{tabular}

\section{UNDERWATER SENSOR NETWORKS APPLICATIONS} networks as:

The described features in the literature enable a broad range of applications for underwater sensor

- Environmental Monitoring: Environmental monitoring is one of the most important applications of underwater sensor networks. Such as Monitoring of chemical and biological pollutions, ocean currents and winds, weather forecasting and detecting climate changes [18].

- Assisted Navigation: Exploring and locating rocks, shoals, mooring positions, submerged wrecks and any other critical position of interest are another important application of underwater sensor networks.

- Distributed Tactical Surveillance: Using underwater sensor networks one can monitor an area for surveillance, reconnaissance, targeting and intrusion detection systems [19].

- Seismic Monitoring of Underwater Fields: Seismic monitoring of underwater fields is another important application of underwater sensor networks. Studies of variation in the oil reservoir over time which can be used for assessment of field performance and necessary interventions refer to the topic of "4-D seismic monitoring".

- Disaster prevention: measuring remote location seismic activity from remote locations can help to detect oceans disaster such as tsunami.

- Cooperative Ocean Sampling Networks: Networks on underwater sensors can be used to perform synoptic, cooperative adaptive sampling of the 3D coastal ocean environment. The advantages of bringing together sophisticated new robotic vehicles and advanced ocean models in improving the ability to observe and predict the oceanic environment have been demonstrated by Monterey Bay Experiment accomplished in August 2003.

- Underwater Mine Detection and Identification: The concurrent operation of multiple sensor networks with acoustic and optical sensors can be used to perform rapid environmental assessments of unknown objects. Therefore, this scheme can facilitate the underwater mine detection and identification.

- Communication between Underwater Robots: Underwater autonomous robots can be controlled through establishing communication by underwater sensor networks. In coordinated with sensor networks applications underwater robots should make communication while they are either fully autonomous or able to communicate, but limited in depth deployment and maneuverability.

\section{UNDERWATER SENSOR NETWORKING CHALLENGES}

UWSN has its own characteristic, for example, underwater acoustic channels are unique. Over all, terrestrial networks specification could not be used in underwater acoustic ones.

\subsection{Physical Implementation Limitations}

Radio or optical methods provide long-distance communication with high bandwidths; therefore the electromagnetic spectrum takes over the communication applications outside water. On the contrary, water 
attenuates and scatters almost waves of all electro-magnetic frequencies, making acoustic waves preferable for underwater communication beyond tens of meters. Fundamental attenuation of all electro-magnetic frequencies in water describes the power loss that a tone at frequency experiences as it travels from one location to another. Acoustic wave's propagation popular frequency range could be described in several stages: In the first stage, it takes over the fundamental loss that occurs in a transmission with distance d. The second stage deals with site specific loss, due to surface-bottom reflections and refraction sound speed changes in depth. The third stage is about addresses that have been randomly changes caused by slow variations in the propagation medium, in the large-scale received power [20].

\subsection{Medium Access Control and Resource Sharing}

Sharing communication resources among nodes can be performed in multi-user systems. In wireless sensor networks, the frequency spectrum is inherently shared and interference needs to be properly managed. Efficient sharing of resources in stations is performed through different methods to separate the signals coexisted in a common medium. In design of resource sharing schemes for underwater networks, the peculiar characteristics of the acoustic channel like the most relevant of which are long delays, frequency-dependent attenuation, and relatively long arrival of acoustic signals should be considered, plus bandwidth constraints of acoustic hardware. Signals can be deterministically separated in time or frequency [21].

\subsection{Reliable Data Transfer}

Another two approaches are mentioned for reliable data transfer. First one is end-to-end technique, the most favorite Transmission Control Protocol (TCP). The second technique is hop-by-hop. Generally TCP's performance may face problem because of high and dynamic channel errors and propagation delay [21].

\subsection{Multi-hop Routing}

Data forwarding efficiently from source nodes to command/control stations could be very challenging, especially in long-term mobile transmission that are saving energy is an important issue. At the same time, routing should be able to handle node mobility. This requirement makes most existing energy efficient routing protocols unsuitable for UWSNs. Various routing protocols are [21],

- Vector based forwarding (VBF)

- Focused beam routing (FBR)

- Reliable and Energy Balanced Routing Algorithm (REBAR)

- Information-Carrying Routing Protocol (ICRP)

- Directional Flooding-Based Routing (DFR)

- Distributed Underwater Clustering Scheme (DUCS)

- Depth Based Routing (DBR)

- Hop-by-Hop Dynamic Addressing Based Routing (H2-DAB)

\subsection{Localization}

Localization of mobile sensor nodes is crucial for underwater sensor networks. For some application such as aquatic monitoring high precision localization is necessary, while localization solution is needed by some applications such as surveillance. However, characteristics of underwater acoustic propagation and mobility of a sensor cause great challenges to high precision and scalable localization solutions, as follows [21]:

- Underwater acoustic channels are highly dispersive and dense multipath impeding delay in arrival estimation time.

- Due to effects of stratification, acoustic signals do not move on a straight path,

- Due to large scale deployment of sensor networks centralized solutions is prevented.

- Sensor mobility entails dynamic network topology change.

\section{DIFFERENCES BETWEEN TERRESTRIAL AND UNDERWATER SENSOR NETWORKS}

The main differences between terrestrial and underwater sensor networks are as follows [2]:

- Cost: Due to complexity and hardware protection challenges underwater sensors unlike terrestrial sensor nodes are expensive.

- Deployment. Due to the cost and challenges associated with the deployment of sensor nodes, compared to terrestrial sensor nodes which are compactly deployed, underwater sensor nodes deployment is sparser. 
- Power. Due to long distances and more complexity of signal processing at the receivers, the power assumption for underwater sensor network communication is higher than radio communication.

- Memory. While underwater sensors carry out some data storage on the other hand, terrestrial sensor nodes have very limited storage capacity.

- Spatial Correlation. Because of farther distance between underwater sensors, readings data is different from readings in terrestrial sensors.

\section{CONCLUSION}

UWSNs have been concerned in recent years for their applications in data collecting, by scattering sensors in water. Underwater wireless sensor networks have important applications in remote monitoring and target tracking, due to their intelligent sensors. Equipping with wireless interfaces with which they communicate raises various problems specified to underwater monitoring, including the communication medium. The design of a UWSN depends on factors such as environment, application's design objectives, and cost, hardware and etc. In this paper, an overview of the recently published papers on the new applications of underwater sensor networks was presented. Some possible challenges on development and implementation of UWSNs were also reviewed and discussed. It has reviewed a comparison between terrestrial and UWSN. This survey will give the readers a view on the place of underwater sensor networks on researches and industries by providing statistical data. The readers can track what have been the highly interest in recent years and what are yet to come.

\section{REFERENCES}

[1] J. Heidemann, et al., "Underwater sensor networks: applications, advances and challenges,” Philosophical Transactions of the Royal Society of London A: Mathematical, Physical and Engineering Sciences, vol/issue: 370(1958), pp. 158-175, 2012.

[2] I. F. Akyildiz, et al., "Underwater acoustic sensor networks: research challenges,” Ad hoc networks, vol/issue: 3(3), pp. 257-279, 2005.

[3] M. Murad, et al., “A Survey on Current Underwater Acoustic Sensor Network Applications,” International Journal of Computer Theory and Engineering, vol/issue: 7(1), pp. 51, 2015.

[4] E. Felemban, et al., "Underwater Sensor Network Applications: A Comprehensive Survey,” International Journal of Distributed Sensor Networks, vol. 501, pp. 896832, 2015.

[5] G. Han, et al., "Impacts of Deployment Strategies on Localization Performance in Underwater Acoustic Sensor Networks,” Industrial Electronics, IEEE Transactions on, vol/issue: 62(3), pp. 1725-1733, 2015.

[6] S. Kundu and P. Sadhukhan, "Design and implementation of a time synchronization-free distributed localization scheme for underwater acoustic sensor network," In Applications and Innovations in Mobile Computing (AIMoC), 2015, pp. 74-80.

[7] Z. Wu and X. Li, “An improved underwater acoustic network localization algorithm,” Communications, China, vol/issue: 12(3), pp. 77-83, 2015

[8] H. Ramezani and G. Leus, "Localization Packet Scheduling for Underwater Acoustic Sensor networks," Selected Areas in Communications, IEEE, vol/issue: 33(7), pp. 1345-1356, 2015.

[9] V. Krishnaswamy and S. S. Manvi, "Analysis of acoustic channel in underwater acoustic sensor network," in Advance Computing Conference (IACC), 2015 IEEE International, pp. 233-236.

[10] D. K. Jha, et al., “Topology optimization for energy management in underwater sensor networks,” International Journal of Control, vol/issue: 88(9), pp. 1775-1788, 2015.

[11] R. Mohammadi, et al., "Fuzzy Depth Based Routing Protocol for Underwater Acoustic Wireless Sensor Networks," Journal of Telecommunication, Electronic and Computer Engineering (JTEC), vol/issue: 7(1), pp. 81-86, 2015.

[12] B. Diao, et al., "Improving Both Energy and Time Efficiency of Depth-Based Routing for Underwater Sensor Networks,” International Journal of Distributed Sensor Networks, vol. 501, pp. 781932, 2015.

[13] R. Su, et al., "Balancing between robustness and energy consumption in underwater acoustic sensor networks," in Wireless Communications and Networking Conference (WCNC), 2015 IEEE, pp. 1048-1053.

[14] G. Han, et al., "Secure communication for underwater acoustic sensor networks," Communications Magazine, IEEE, vol/issue: 53(8), pp. 54-60, 2015.

[15] K. Hao, et al., "An Efficient and Reliable Geographic Routing Protocol Based on Partial Network Coding for Underwater Sensor Networks,” Sensors, vol/issue: 15(6), pp. 12720-12735, 2015.

[16] L. Karim, et al., "Localization in terrestrial and underwater sensor-based $\mathrm{m} 2 \mathrm{~m}$ communication networks: architecture, classification and challenges,” International Journal of Communication Systems, 2015. DOI: 10.1002/dac.2997.

[17] S. D. Seeley Jr. and R. Balasubramanian, "GEMMP - A Google Maps Enabled Mobile Mission Planning Tool for Autonomous Underwater Vehicles,” International Journal of Robotics and Automation (IJRA), vol/issue: 1(2), pp. 94 106, 2012. ISSN: 2089-4856.

[18] H. Mei, et al., "Joint Interference Mitigation with Channel Estimated in Underwater Acoustic System," TELKOMNIKA, vol/issue: 11(12), pp. 7423 7430, 2013. e-ISSN: 2087-278X. 
[19] J. Heidemann, et al., "Research challenges and applications for underwater sensor networking," in Wireless Communications and Networking Conference, 2006. WCNC 2006. IEEE, vol. 1, pp. 228-235, 2006.

[20] R. B. Manjula. and S. M. Sunilkumar, "Issues in Underwater Acoustic Sensor Networks," International Journal of Computer and Electrical Engineering, vol/issue: 3(1), pp. 1793-8163, 2011.

[21] I. F. Akyildiz, et al., "A survey on wireless multimedia sensor networks," Computer networks, vol/issue: 51(4), pp. 921-960, 2007.

[22] Manigopal A. and Panneerselvam R., "Underwater wireless sensor networks: a survey,” International Journal of Computer Science and Information Technology \& Security (IJCSITS), vol/issue: 2(6), 2012. ISSN: 2249-9555.

[23] I. F. Akyildiz, et al., "State-of-the-art in protocol research for underwater acoustic sensor networks," in Proceedings of the 1st ACM international workshop on Underwater networks, 2006, September, pp. 7-16. 